\title{
金 - 硫团簇负离子组成特征的探讨*
}

\author{
梁汉东刘敦一 1 \\ (煤炭资源教育部重点实验室, 中国矿业大学北京校区，北京 100083 ; \\ ${ }^{1}$ 国土资源部同位素地质开放实验室, 中国地质科学院, 北京 100037)
}

\begin{abstract}
摘要 报导了金/硫靶在静态二次离子质谱实验条件下形成的组成通式为 $\mathrm{Au}_{1-15} \mathrm{~S}_{0-5}$ 的团簇负离子. 其中, $\mathrm{Au}_{n}$ 组成系列具有典型的奇数优势, 属于金的一元团簇负离子. 其余组成系列 $\left(\mathrm{Au}_{1-15} \mathrm{~S}_{1-5}\right)$ 则属于金 - 硫二元团簇负 离子. 研究发现, 金 - 硫团簇负离子具有以下与组成密切相关的特征: (1) 它们继承了金一元团簇负离子的奇数 优势; (2) 它们中硫原子数仅限于 5 , 而金原子数则在较大范围变化; (3) 它们完全没有多数其它金属与非金属(包 括硫)二元团簇正/负离子共有的 $\mathrm{Me}_{n} \mathrm{~N}_{n}$ 型组成优势; (4) 它们以上组成特征及其它细节恰似碳 - 磷这样的典型 非金属二元团簇正/负离子. 基于这样的组成特征可提出, 金 - 硫团簇负离子在构成上包含着相对完整的金一元 团簇组件, 在形成机制上源于金一元团簇与硫一元团簇 (或称为: 限于五原子的多硫单元)之间的再团簇化. 关于 再团簇化机制中的相互作用问题, 即两类团簇间是依靠末端原子交叉成键还是依赖两类团簇中异种原子之间静 电诱导力等“非共价键”作用的问题, 则有待于今后研究证实.
\end{abstract}

关键词：金 - 硫团簇，金团簇，五硫单元，再团簇化，团簇负离子，组成特征，静态二次离子质谱

近十多年来, 物质气相团簇化学的质谱实验研 究蓬勃兴起, 尤其在金属与非金属的二元团簇离子 研究方面取得许多进展 ${ }^{[1-7]}$. 关于金属 $(\mathrm{Me})$ 与硫 $(\mathrm{S})$ 的二元团簇离子的研究已涉及到 $\mathrm{Fe} 、 \mathrm{Mn} 、 \mathrm{Co}$ 、 $\mathrm{Ni} 、 \mathrm{Zn} 、 \mathrm{Cu} 、 \mathrm{~Pb}$ 等众多常见金属 ${ }^{[8-10]}$, 但金与硫的二 元团簇离子至今没有报导. 然而, 金的一元团簇 正/负离子却早有观测 ${ }^{[11,12]}$. Becker 及其合作者 ${ }^{[13]}$ 的研究发现金团簇离子具有显著的“幻数效应 (magic number)”, 亦即, 具有奇数金原子个数的金 团簇离子质谱峰在强度上占相对优势. 最新的研 究表明 ${ }^{[14]}$, 金团簇 (单电荷) 离子序列的奇数优势在 金团簇双电荷离子 $\left(\mathrm{Au}_{n}^{2+}\right)$ 序列中则反转, 在三电荷 离子 $\left(\mathrm{Au}_{n}^{3+}\right)$ 序列中趋于消失。在团簇离子实验方 法上, 多数工作是运用配置激光束溅射/电离的飞 行时间 (TOF) 质谱或离子回旋共振 (ICR) 质谱. 以 离子束溅射/电离为特点的二次离子质谱 (SIMS) 虽 然主要用于高纯材料分析, 但有时也被用于研究团 簇离子 ${ }^{[12]}$. 过去在关于 SIMS 的离子束溅射机理的
研究中就观测到在使用重原子惰性气体离子束 $\left(\mathrm{Xe}^{+}\right)$时产生的硅、铝、铜、碳纯元素的团簇离子 $\left(\mathrm{Si}_{n}^{+}, \mathrm{Al}_{n}^{+} \text {和 } \mathrm{Al}_{n}^{2,3+}, \mathrm{Cu}_{n}^{+}, \mathrm{C}_{n}^{-}\right)^{[15]}$, 现在看来 $\mathrm{SIMS}$ 中 形成的团簇离子与激光质谱中的结果并没有实质差 别. 例如: SIMS 中铜团簇离子同样出现奇数优势 ${ }^{[15]}$. 作者 ${ }^{[16]}$ 曾采用配镓离子束的飞行时间型二次离子 质谱(TOF SIMS) 初步研究了无机元素之间以及与 有机物种之间的表面相化学反应; 还观测到源于硫 的组成为 $S_{1-12}$ 的负离子系列 ${ }^{[17]}$. 总之, SIMS 用于 团簇离子的观测和研究在实验上具有可行性, 是值 得探索的工作. 本文将报导运用 TOF SIMS 观察到 的金 - 硫团簇负离子的结果, 并着重考察其组成规 律.

\section{1 实验与仪器}

\section{1 样品靶制备}

取 $0.2 \mathrm{~g}$ 硫 [纯度 $99.998 \%$ (质量分数, 下同), Ardri 化学试剂公司]溶解于 $25 \mathrm{~mL}$ 正己烷. 用微量 
进样头取约 $0.2 \mu \mathrm{L}$ 溶液分别点滴于金箔(纯度 99. 999\%, Ardri 化学试剂公司), 并让液滴在表面上 自然充分扩散并初步风干后, 将这样制备出的 $\mathrm{S}$ / $\mathrm{Au}$ 样品靶送人仪器进样池低真空 $\left(10^{-2} \mathrm{~Pa}\right)$ 区进一 步风干 $10 \mathrm{~min}$, 随后进人仪器高真空 $\left(\sim 2.5 \times 10^{-8}\right.$ $\mathrm{Pa}$ )样品分析室待测。样品靶的制备和随后的仪器 实验分别在 100 级洁净台和 100 级洁净工作间内进 行。对照实验采用铜簿(纯度 $99.99 \%$, Ardri 化学 试剂公司) 代替金䈃。将上述硫溶液取 $0.2 \mu \mathrm{L}$ 点滴 于铜䇴表面, 制成 $\mathrm{S} / \mathrm{Cu}$ 对照靶, 其它步骤与上述相 同.

\section{2 仪器与实验条件}

主要仪器是高性能静态飞行时间型二次离子 质谱仪 ${ }^{[18,19]}$ (TFS-2000, LMIG TOF SIMS, 美国 PHIEvans)。采用液态金属镓 $\left({ }^{69} \mathrm{Ga}^{+}\right)$束电离源。束电流 $1 \mu \mathrm{A}$, 束电压 $15 \mathrm{kV}$, 扫描微区 $2 \mu \mathrm{m}$, 扫描时间 6 min. 样品室真空优于 $2.5 \times 10^{-8} \mathrm{~Pa}$. 实验时标定的 质量分辨为 $4000\left({ }^{28} \mathrm{Si}\right)$ 或优于 $10000\left({ }^{197} \mathrm{Au}\right)$. 由此 质量分辨率可见, TFS-2000 型二次离子质谱具有准 高分辨本领, 质量 $(m)$ 测定通常能够在 $2000 \mathrm{amu}$ 以下给出含两位小数的有效数值. 另外, 质谱峰的 强度 $(I)$ 通常以离子计数 (ion counts) 表达。质量采 集范围可达 $10000 \mathrm{amu}$.

\section{2 结果和讨论}

\section{1 实验结果}

图 1 是实验获得的局部质谱图。图中显示了三
组典型的团簇负离子峰, 对应的化学组成分别为: $\mathrm{Au}_{3} \mathrm{~S}_{0-5}(m / z$ 591, 623, 655, 587, 719 和相对丰度很 小的 $m / z 751$ (见图 1 中质谱数据系统自动标识 峰 $m / z 750$ 右侧的小峰 ) ); $\mathrm{Au}_{5} \mathrm{~S}_{0-5}(m / z$ 985, 1017 , $1049,1081,1113$ 和 $m / z 1145) ; \mathrm{Au}_{7} \mathrm{~S}_{0-5}(m / z 1379$, $1411,1$ 443, 1 475, 1507 和 $m / z 1539)$.

实验检出的团簇负离子相当丰富, 从最低系列 $\mathrm{AuS}_{0-5}(m / z$ 197, 229, 261, 293, 325 和 $m / z$ 357), 到 相当高质量系列 $\mathrm{Au}_{15} \mathrm{~S}_{0-5}(m / z) 2955,2$ 987, 3019 , 3050,3082 和 $m / z 3$ 114). 全部团簇负离子质谱峰 的实测质量 $(m)$ 和强度 $(I)$ 与确定的对应化学组成 数据汇集于表 1 . 总体上, 观测到的团簇负离子的组 成通式可表达为 $\mathrm{Au}_{1-15} \mathrm{~S}_{0-5}$. 或者, 更确切地表达为 $\mathrm{Au}_{n}$ 和 $\mathrm{Au}_{n} \mathrm{~S}_{1 \sim 5}$, 前者属于一元金团簇负离子的组 成, 后者则是真正意义上的二元金 - 硫团簇负离子 的组成.

\section{2 金 - 硫团簇离子组成特征的探讨}

本文利用 $\mathrm{S} / \mathrm{Cu}$ 靶在与 $\mathrm{S} / \mathrm{Au}$ 靶相同样品处理 和相同仪器条件下的实验结果汇集于表 2 . 显而易 见，化学组成上铜与硫原子比符合经典化学成键的 理论比值 $(1: 1)$ 的铜 - 硫团簇 $\left(\mathrm{Cu}_{n} \mathrm{~S}_{n}\right)$ 负离子在强度 上占绝对优势 (见表 2 中的组成与强度数据). 在团 簇物种与组成的关系上也限于单个原子的差异. 实 验观测到 $\mathrm{Cu}_{n} \mathrm{~S}_{n+1}$ 和 $\mathrm{Cu}_{n} \mathrm{~S}_{n-1}$, 却未检出 $\mathrm{Cu}_{n} \mathrm{~S}_{n+2}$ 或 $\mathrm{Cu}_{n} \mathrm{~S}_{n-2}$. 实际上, 铜 - 硫团簇离子的优势组成特 点在许多其它金属元素与非金属元素二元团簇离子 中也得到普遍反映. Shi 和 Liu 等 ${ }^{[9,10]}$ 采用激光/质

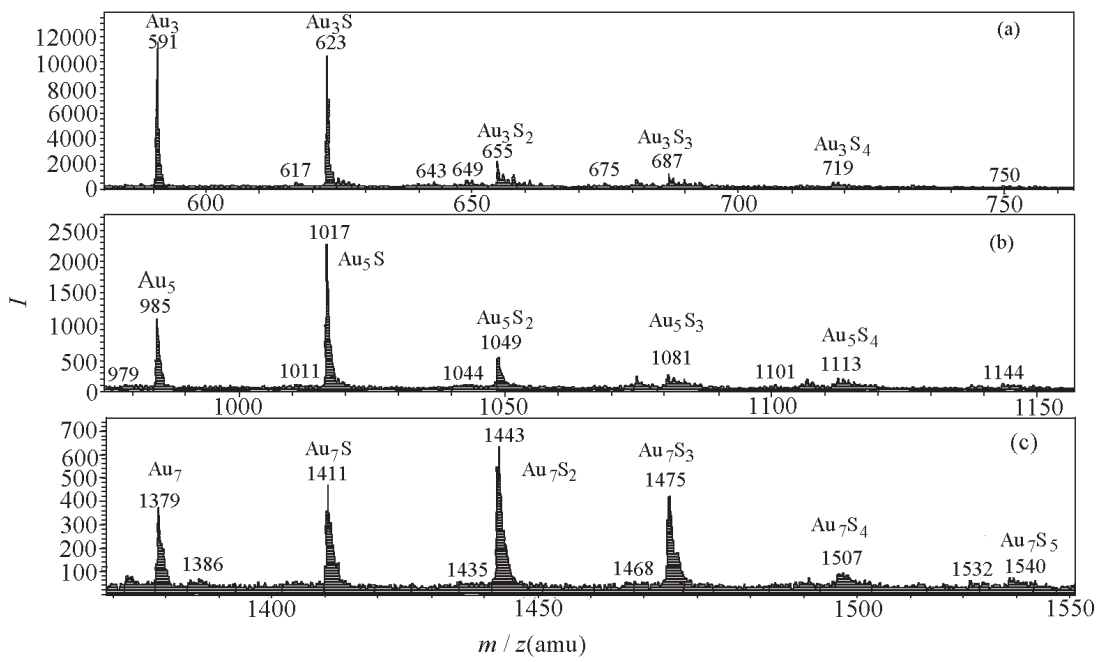

图 1 来自 $\mathrm{S} / \mathrm{Au}$ 靶的团簇负离子的局部质谱图

Fig. 1 A part of negative ion mass spectra of cluster derived from $\mathrm{S} / \mathrm{Au}$ target cluster ions with $\mathrm{Au}_{3} \mathrm{~S}_{0-5}$ (a), with $\mathrm{Au}_{5} \mathrm{~S}_{0-5}$ (b), with $\mathrm{Au}_{7} \mathrm{~S}_{0-5}$ (c), ion counts (I) 
表 1 组成为 $\mathrm{Au}_{1-15} \mathrm{~S}_{0-5}$ 的团簇负离子实验数据

Table 1 Data for the cluster negative ions with formula of $\mathrm{Au}_{1-15} \mathrm{~S}_{0-5}$

\begin{tabular}{|c|c|c|c|c|c|c|c|c|c|c|c|}
\hline Comp. & $\mathrm{m} / \mathrm{amu}$ & $I$ & Comp. & $\mathrm{m} / \mathrm{amu}$ & $I$ & Comp. & $m / \mathrm{amu}$ & $I$ & Comp. & $\mathrm{m} / \mathrm{amu}$ & $I$ \\
\hline $\mathrm{Au}$ & 197.96 & 384273 & $\mathrm{Au}_{2}$ & 393.92 & 30211 & $\mathrm{Au}_{3}$ & 590.89 & 30488 & $\mathrm{Au}_{4}$ & 787. 73 & 3589 \\
\hline $\mathrm{AuS}$ & 228.95 & 60123 & $\mathrm{Au}_{2} \mathrm{~S}$ & 425.90 & 40133 & $\mathrm{Au}_{3} \mathrm{~S}$ & 622.86 & 29390 & $\mathrm{Au}_{4} \mathrm{~S}$ & 819. 69 & 4636 \\
\hline $\mathrm{AuS}_{2}$ & 260.94 & 20577 & $\mathrm{Au}_{2} \mathrm{~S}_{2}$ & 457. 88 & 13608 & $\mathrm{Au}_{3} \mathrm{~S}_{2}$ & 654.83 & 5878 & $\mathrm{Au}_{4} \mathrm{~S}_{2}$ & 851.69 & 8853 \\
\hline $\mathrm{AuS}_{3}$ & 292.89 & 9941 & $\mathrm{Au}_{2} \mathrm{~S}_{3}$ & 489. 84 & 821 & $\mathrm{Au}_{3} \mathrm{~S}_{3}$ & 686.75 & 4. 323 & $\mathrm{Au}_{4} \mathrm{~S}_{3}$ & 883. 68 & 2126 \\
\hline $\mathrm{AuS}_{4}$ & 324.86 & 8875 & $\mathrm{Au}_{2} \mathrm{~S}_{4}$ & 521.80 & 870 & $\mathrm{Au}_{3} \mathrm{~S}_{4}$ & 718.76 & 1696 & $\mathrm{Au}_{4} \mathrm{~S}_{4}$ & 915.66 & 860 \\
\hline $\mathrm{AuS}_{5}$ & 356.86 & 3741 & $\mathrm{Au}_{2} \mathrm{~S}_{5}$ & 553.75 & 643 & $\mathrm{Au}_{3} \mathrm{~S}_{5}$ & 750.72 & 1018 & $\mathrm{Au}_{4} \mathrm{~S}_{5}$ & 947.58 & 491 \\
\hline $\mathrm{Au}_{5}$ & 984.78 & 4840 & $\mathrm{Au}_{6}$ & 1181.69 & 1503 & $\mathrm{Au}_{7}$ & 1378.77 & 1985 & $\mathrm{Au}_{8}$ & 1575.62 & 411 \\
\hline $\mathrm{Au}_{5} \mathrm{~S}$ & 1016.73 & 9486 & $\mathrm{Au}_{6} \mathrm{~S}$ & 1213.59 & 575 & $\mathrm{Au}_{7} \mathrm{~S}$ & 1410.72 & 2386 & $\mathrm{Au}_{8} \mathrm{~S}$ & 1607.60 & 372 \\
\hline $\mathrm{Au}_{5} \mathrm{~S}_{2}$ & 1048.70 & 3489 & $\mathrm{Au}_{6} \mathrm{~S}_{2}$ & 1245.64 & 1728 & $\mathrm{Au}_{7} \mathrm{~S}_{2}$ & 1442.68 & 3481 & $\mathrm{Au}_{8} \mathrm{~S}_{2}$ & 1639.58 & 326 \\
\hline $\mathrm{Au}_{5} \mathrm{~S}_{3}$ & 1080.65 & 1203 & $\mathrm{Au}_{6} \mathrm{~S}_{3}$ & 1277.55 & 1625 & $\mathrm{Au}_{7} \mathrm{~S}_{3}$ & 1474.72 & 2531 & $\mathrm{Au}_{8} \mathrm{~S}_{3}$ & -- & -- \\
\hline $\mathrm{Au}_{5} \mathrm{~S}_{4}$ & 1112.64 & 616 & $\mathrm{Au}_{6} \mathrm{~S}_{4}$ & 1309.56 & 573 & $\mathrm{Au}_{7} \mathrm{~S}_{4}$ & 1506.66 & 385 & $\mathrm{Au}_{8} \mathrm{~S}_{4}$ & -- & -- \\
\hline $\mathrm{Au}_{5} \mathrm{~S}_{5}$ & 1144.58 & 300 & $\mathrm{Au}_{6} \mathrm{~S}_{5}$ & 1341.51 & 357 & $\mathrm{Au}_{7} \mathrm{~S}_{5}$ & 1538.63 & 387 & $\mathrm{Au}_{8} \mathrm{~S}_{5}$ & 1735.53 & 306 \\
\hline $\mathrm{Au}_{9}$ & 1772.84 & 749 & $\mathrm{Au}_{10}$ & -- & -- & $\mathrm{Au}_{11}$ & 2166.95 & 363 & $\mathrm{Au}_{12}$ & 2364 & + \\
\hline $\mathrm{Au}_{9} \mathrm{~S}$ & 1804.71 & 1287 & $\mathrm{Au}_{10} \mathrm{~S}$ & 2002 & + & $\mathrm{Au}_{11} \mathrm{~S}$ & 2198.79 & 563 & $\mathrm{Au}_{12} \mathrm{~S}$ & 2395.84 & 277 \\
\hline $\mathrm{Au}_{9} \mathrm{~S}_{2}$ & 1836.65 & 1109 & $\mathrm{Au}_{10} \mathrm{~S}_{2}$ & -- & -- & $\mathrm{Au}_{11} \mathrm{~S}_{2}$ & 2230.61 & 596 & $\mathrm{Au}_{12} \mathrm{~S}_{2}$ & 2428 & + \\
\hline $\mathrm{Au}_{9} \mathrm{~S}_{3}$ & 1 868. 58 & 972 & $\mathrm{Au}_{10} \mathrm{~S}_{3}$ & 2066 & + & $\mathrm{Au}_{11} \mathrm{~S}_{3}$ & 2263.84 & 411 & $\mathrm{Au}_{12} \mathrm{~S}_{3}$ & 2460 & + \\
\hline $\mathrm{Au}_{9} \mathrm{~S}_{4}$ & 1900.67 & 499 & $\mathrm{Au}_{10} \mathrm{~S}_{4}$ & 2098 & + & $\mathrm{Au}_{11} \mathrm{~S}_{4}$ & 2294.52 & 318 & $\mathrm{Au}_{12} \mathrm{~S}_{4}$ & -- & -- \\
\hline $\mathrm{Au}_{9} \mathrm{~S}_{5}$ & 1932.82 & 333 & $\mathrm{Au}_{10} \mathrm{~S}_{5}$ & 2130 & + & $\mathrm{Au}_{11} \mathrm{~S}_{5}$ & 2327 & + & $\mathrm{Au}_{12} \mathrm{~S}_{5}$ & -- & -- \\
\hline $\mathrm{Au}_{13}$ & 2561 & 250 & $\mathrm{Au}_{14}$ & -- & -- & $\mathrm{Au}_{15}$ & 2955 & + & $\mathrm{Au}_{16}$ & -- & -- \\
\hline $\mathrm{Au}_{13} \mathrm{~S}$ & 2593 & 409 & $\mathrm{Au}_{14} \mathrm{~S}$ & -- & -- & $\mathrm{Au}_{15} \mathrm{~S}$ & 2987 & + & $\mathrm{Au}_{16} \mathrm{~S}$ & -- & -- \\
\hline $\mathrm{Au}_{13} \mathrm{~S}_{2}$ & 2652 & 382 & $\mathrm{Au}_{14} \mathrm{~S}_{2}$ & -- & -- & $\mathrm{Au}_{15} \mathrm{~S}_{2}$ & 3019 & + & $\mathrm{Au}_{16} \mathrm{~S}_{2}$ & -- & -- \\
\hline $\mathrm{Au}_{13} \mathrm{~S}_{3}$ & 2657 & 291 & $\mathrm{Au}_{14} \mathrm{~S}_{3}$ & -- & -- & $\mathrm{Au}_{15} \mathrm{~S}_{3}$ & 3050 & + & $\mathrm{Au}_{16} \mathrm{~S}_{3}$ & -- & -- \\
\hline $\mathrm{Au}_{13} \mathrm{~S}_{4}$ & 2689 & 271 & $\mathrm{Au}_{14} \mathrm{~S}_{4}$ & -- & -- & $\mathrm{Au}_{15} \mathrm{~S}_{4}$ & 3082 & + & $\mathrm{Au}_{16} \mathrm{~S}_{4}$ & -- & -- \\
\hline $\mathrm{Au}_{13} \mathrm{~S}_{5}$ & 2720 & 188 & $\mathrm{Au}_{14} \mathrm{~S}_{5}$ & -- & -- & $\mathrm{Au}_{15} \mathrm{~S}_{5}$ & 3114 & + & $\mathrm{Au}_{16} \mathrm{~S}_{5}$ & -- & -- \\
\hline
\end{tabular}

Note: The ion counts of the peak, remarked as " +", is a few to be distinguished while that remarked as "--" is very few not to be distinguished.

I: ion counts

谱研究金属与硫二元团簇离子的结果显示, 组成符 合 $\mathrm{Me}_{n} \mathrm{~S}_{n}(\mathrm{Me}=\mathrm{Fe}, \mathrm{Co}, \mathrm{Zn}, \mathrm{Mn}, \mathrm{Pb})$ 的团簇离子占绝 对优势, $\mathrm{Me}_{n} \mathrm{~S}_{n-1}$ 或 $\mathrm{Me}_{n} \mathrm{~S}_{n+1}$ 次之. 陈云清等 ${ }^{[20]}$ 采 用紫外激光光解 (电离) $\mathrm{Se} / \mathrm{Fe}$ 靶产生的 $\mathrm{Fe}-\mathrm{Se}$ 二元 团簇离子优势组成是 $\mathrm{Fe}_{n} \mathrm{Se}_{n}$, 其次是 $\mathrm{Fe}_{n} \mathrm{Se}_{n \pm 1}$. 然 而, 本文所观察到的团簇负离子显然不具备这 种优势组成特点. 图 1 显示: 含 3 个金原子的团簇 负离子其优势组成并不是 $\mathrm{Au}_{3} \mathrm{~S}_{3}(m / z$ 686. 75$)$; 含 7 个金原子的团簇负离子优势组成却为 $\mathrm{Au}_{7} \mathrm{~S}_{2}(\mathrm{~m} / z$ $1442.68)$; 含 13 个金原子的团簇负离子仅以 $\mathrm{Au}_{13} \mathrm{~S}_{1}(m / z 2593$ ) 略占优势 (参见表 1). 实际上, 本 文所观察到的团簇负离子的显著组成特征首先表现
为, 硫原子数与金原子的增长没有直接关系. 团簇 负离子中的金原子数无论是 $7 、 11$ 或 15 , 硫原子数 却保持不大于 5 .

以 $\mathrm{Au}_{n}$ 为组成的系列团簇负离子呈现出明显 的奇数优势, 亦即, 含奇原子数的团簇离子其丰度 (I) 相对较高 (参见图 2). 这种奇数优势在其它一元 团簇研究中经常可见. 例如, 纯碳的团簇 $\left(\mathrm{C}_{n}\right)$ 离子 在低质量端 $(n<30)$ 普遍存在奇数优势 ${ }^{[21]}$, 它被认 为对应着低碳数 (中性) 小团簇的亚稳状态, 并且与 稳定态大团簇物质 (如富勒烯, $\mathrm{C}_{60} / \mathrm{C}_{70}$ ) 的最终形成 有着密切关系 ${ }^{[22]}$. 鉴于 $\mathrm{Au}_{n}$ 系列负离子奇数优势 的存在, 作者认为本文实验检测到的 $\mathrm{Au}_{n}$ 系列负离 
表 2 从 $\mathrm{S} / \mathrm{Cu}$ 靶检出的全部 $\mathrm{Cu}-\mathrm{S}$ 团簇负离子的实测质量和强度数据

Table 2 Data for $\mathrm{Cu}-\mathrm{S}$ cluster negative ions derived from $\mathrm{S} / \mathrm{Cu}$ target

\begin{tabular}{|c|c|c|c|c|c|c|c|c|}
\hline Comp. & $m / \mathrm{amu}$ & $I$ & Comp. & $m / \mathrm{amu}$ & $I$ & Comp. & $m / \mathrm{amu}$ & $I$ \\
\hline${ }^{63} \mathrm{CuS}$ & 94.92 & 27966 & ${ }^{63} \mathrm{Cu}_{2} \mathrm{~S}_{2}$ & 189.84 & 1663 & ${ }^{63} \mathrm{Cu}_{3} \mathrm{~S}_{3}$ & 284.75 & 257 \\
\hline \multirow[t]{3}{*}{${ }^{65} \mathrm{CuS}$} & 96.91 & 18406 & ${ }^{65} \mathrm{Cu}^{63} \mathrm{CuS}_{2}$ & 191.85 & 1668 & ${ }^{63} \mathrm{Cu}_{2}{ }^{65} \mathrm{CuS}_{3}$ & 286.75 & 351 \\
\hline & & & ${ }^{65} \mathrm{Cu}_{2} \mathrm{~S}_{2}$ & 193.84 & 519 & ${ }^{63} \mathrm{Cu}^{65} \mathrm{Cu}_{2} \mathrm{~S}_{3}$ & 288. 79 & 212 \\
\hline & & & & & & ${ }^{65} \mathrm{Cu}_{3} \mathrm{~S}_{3}$ & 290.85 & 88 \\
\hline${ }^{63} \mathrm{CuS}_{2}$ & 126.89 & 12108 & ${ }^{63} \mathrm{Cu}_{2} \mathrm{~S}_{3}$ & 211.85 & 443 & ${ }^{63} \mathrm{Cu}_{3} \mathrm{~S}_{4}$ & 316.75 & 34 \\
\hline \multirow[t]{3}{*}{${ }^{65} \mathrm{CuS}_{2}$} & 128.90 & 6153 & ${ }^{63} \mathrm{Cu}^{65} \mathrm{CuS}_{3}$ & 223.82 & 454 & ${ }^{63} \mathrm{Cu}_{2}{ }^{65} \mathrm{CuS}_{4}$ & 318. 70 & 39 \\
\hline & & & ${ }^{65} \mathrm{Cu}_{2} \mathrm{~S}_{3}$ & 225.83 & 150 & ${ }^{63} \mathrm{Cu}^{65} \mathrm{Cu}_{2} \mathrm{~S}_{4}$ & 320.71 & 39 \\
\hline & & & & & & ${ }^{65} \mathrm{Cu}_{3} \mathrm{~S}_{4}$ & 322.73 & 20 \\
\hline${ }^{63} \mathrm{Cu}_{2} \mathrm{~S}$ & 157.87 & 103 & ${ }^{63} \mathrm{Cu}_{3} \mathrm{~S}_{2}$ & 252.81 & 67 & ${ }^{63} \mathrm{Cu}_{4} \mathrm{~S}_{3}$ & 347 & small \\
\hline${ }^{65} \mathrm{Cu}^{63} \mathrm{CuS}$ & 159.87 & 105 & ${ }^{63} \mathrm{Cu}_{2}{ }^{65} \mathrm{CuS}_{2}$ & 254.79 & 84 & ${ }^{63} \mathrm{Cu}_{3}{ }^{65} \mathrm{CuS}_{3}$ & 349 & small \\
\hline \multirow[t]{3}{*}{${ }^{65} \mathrm{Cu}_{2} \mathrm{~S}$} & 161.86 & 39 & ${ }^{63} \mathrm{Cu}^{65} \mathrm{Cu}_{2} \mathrm{~S}_{2}$ & 256.80 & 64 & ${ }^{63} \mathrm{Cu}_{2}{ }^{65} \mathrm{Cu}_{2} \mathrm{~S}_{3}$ & 351 & small \\
\hline & & & ${ }^{65} \mathrm{Cu}_{3} \mathrm{~S}_{2}$ & 258.83 & small & ${ }^{65} \mathrm{Cu}^{65} \mathrm{Cu}_{3} \mathrm{~S}_{3}$ & 353 & small \\
\hline & & & & & & ${ }^{65} \mathrm{Cu}_{4} \mathrm{~S}_{4}$ & 355 & n. d. \\
\hline
\end{tabular}

I: ion counts

子可能主要不是来自 $\mathrm{Au}_{n} \mathrm{~S}_{1-5}$ 系列团簇负离子的质 谱裂分的贡献, 而是来源于 $\mathrm{Au} / \mathrm{S}$ 靶中金的直接溅 射并成团簇化. 这也就是说, $\mathrm{Au}_{n}$ 系列团簇负离子 指示的是金的独立团簇.

金 - 硫团簇 $\left(\mathrm{Au}_{1-15} \mathrm{~S}_{1-5}\right)$ 负离子明显继承了金 团簇负离子的奇数优势. 如图 3 所示, 无论含单个 硫原子的团簇离子强度 (图 $3 \mathrm{a}$ ), 还是含 1 到 5 个硫 原子的团簇离子强度之和 (图 $3 \mathrm{~b}$ ), 总体上呈现出相 对于金原子数的奇数优势. 类似的现象在最近曾嵘 等 ${ }^{[23]}$ 采用激光束辐射石墨与磷 $\left(\mathrm{P}_{4}\right)$ 的实验结果中也 存在 . 那里, 碳 - 磷 $(\mathrm{C} / \mathrm{P})$ 二元团簇离子同样明显 继承了碳团簇离子在低碳数区域的奇数优势。其

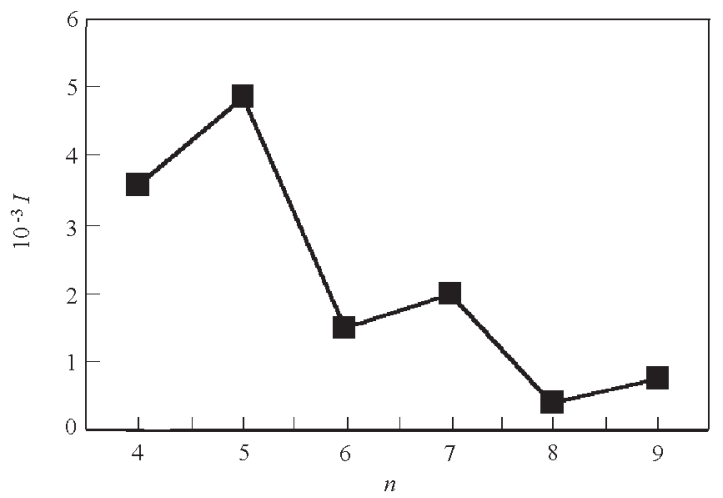

图 2 不同金原子数 $(n)$ 时金团簇负离子强度 $(I)$

Fig. 2 Intensity ( $I)$ of $\mathrm{Au}_{n}$ with number ( $n$ ) of gold atom

$I$ : ion counts
实, 金 - 硫团簇负离子与碳 - 磷团簇正/负离子之间 在现象上还有更多的可比性, 尽管在称谓上前者属 于金属与非金属二元团簇, 后者则是典型的非金属 与非金属二元团簇 . 前者金原子数目在较大范围内 变化, 而硫原子数目是限定的 $(1,2,3,4,5)$; 后者碳 原子数目在大尺度上变化, 而磷原子数目也是限定 的 $(1,2,3,5$, 缺 4$)$; 与此有关的是两者均不具有许 多金属 - 非金属 (包括硫)二元团簇离子常有的那种 $\mathrm{Me}_{n} \mathrm{~N}_{n}$ 型组成优势。在结构上, 曾嵘等 ${ }^{[23]}$ 对含两个 磷原子和低碳数的团簇 $\left(\mathrm{C}_{3-9} \mathrm{P}_{2}\right)$ 作了解释, 认为磷

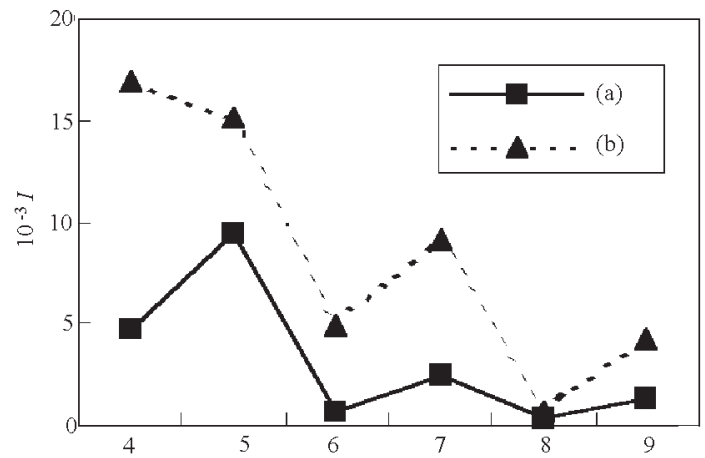

图 3 不同金原子数 $(n)$ 时金 - 硫团簇负离子强度 $(I)$

Fig. 3 Intensity ( $I$ ) of $\mathrm{Au}_{n} \mathrm{~S}_{1-5}$ with number $(n)$ of gold atom

a) Intensity of single series of $\mathrm{Au}_{n} \mathrm{~S}_{1}$

b) total intensity of five series of $\mathrm{Au}_{n} \mathrm{~S}_{1,2,3,4,5}$

$I$ : ion counts 
原子可能在碳链 $\left(\mathrm{C}_{n}\right)$ 的末端成键, 如生成 $\mathrm{PPC}_{6}$ 或 $\mathrm{PC}_{6} \mathrm{P}$, 或者磷双原子单元 $(\mathrm{PP})$ 与碳链的末端成键并 构成环状结构。这样的成键方式和结构意味着在碳 - 磷团簇结构中必然存在着纯碳团簇 $\left(\mathrm{C}_{n}\right)$ 组件; 在 微观形成机制上也必然是碳以团簇整体形式 $\left(\mathrm{C}_{n}\right)$ 与磷原子单元 $\left(\right.$ 如 $\mathrm{P}_{2}$ ) 结合.

通过上述比较可认为, 金 - 硫团簇 $\left(\mathrm{Au}_{1-15} \mathrm{~S}_{1-5}\right)$ 负离子的结构中很可能同样存在完整的纯金原子团 簇 $\left(\mathrm{Au}_{1-15}\right)$, 在微观形成机制上它同样可能源于金 原子团簇 $\left(\mathrm{Au}_{1-15}\right)$ 与多硫原子单元 $\left(\mathrm{S}_{1-5}\right)$ 之间的相 互作用. 多硫原子单元亦可视为原子数目受限定的 硫原子团簇 . 因此, 又可以认为金 - 硫团簇负离子 的来源与金团簇与硫团簇这两类团簇之间进一步团 簇化密切相关. 关于维系金和硫这两个团簇的相互 作用性质, 尚有待进一步研究。事实上, 碳 - 磷团簇 离子中的大部分成键方式和结构问题, 如含三个和 五个磷原子的团簇内部碳磷成键和结构问题, 以及 缺失含四个磷原子的碳 - 磷团簇问题等, 同样还没 有得到解释 ${ }^{[23]}$.

综上所述, 金 - 硫团簇负离子在组成特征上与 大多数金属 - 非金属二元团簇正/负离子有着明显 差别. 金 - 硫团簇负离子与作为非金属与非金属的 碳 - 磷二元团簇正/负离子的组成特征却十分相 似. 相似性的实质在于此两类二元团簇离子内部均 包含有相对完整的一元团簇组件. 把握这一实质对 深人理解团簇问题富有价值 .

至此, 结合前人的研究成果, 能够作如下推论: (1) A 和 B 两种元素的二元团簇 (离子)在微观形成 机制上至少存在两条途径。第一类是 $\mathrm{A}$ 原子与 $\mathrm{B}$ 原子之间优先键合然后再团簇化. 此类最终呈现出 $\mathrm{A}_{n} \mathrm{~B}_{n}$ 组成优势, 该组成优势亦能够表达为 $(\mathrm{AB})_{n}$, 次 优势同理可以表达为 $\mathrm{A}(\mathrm{AB})_{n}$ 或 $(\mathrm{AB})_{n} \mathrm{~B}$. 第二类是 $\mathrm{A}$ 和 $\mathrm{B}$ 中的一方或双方首先并没有 (从固态) 过渡 到游离原子态, 因此不具备 $\mathrm{A}$ 原子与 $\mathrm{B}$ 原子优先键 合的条件; 相反, $\mathrm{A}$ 和 B 中的一方或双方优先独立 成为一元团簇, 然后进一步团簇化形成新的二元团 簇. 理论上可以预计后者可分为两种模式: $\mathrm{A}$ 团簇 与 $\mathrm{B}$ 原子作用; $\mathrm{A}$ 团簇与 $\mathrm{B}$ 团簇作用. 还可以预计 第二类团簇 (离子)中的化学相互作用可能出现更丰 富多彩的方式: A 团簇结构末端与 $\mathrm{B}$ 原子或 B 团簇 末端原子间形成完整化学键, 如共价键; $\mathrm{A}$ 团簇与 $\mathrm{B}$ 团簇以静电诱导力等非共价键, 或以微观层次上的
表面或界面相互作用等值得探讨的方式．(2)金属 与非金属, 非金属与非金属, 乃至金属与金属间的多 元团簇 (离子) 在组成、结构和形成机制上并不存在 严格的界限。

作为金属与非金属的金 - 硫团簇负离子与作为 非金属与非金属的碳 - 磷团簇正/负离子在组成和 结构上的相似。看似巧合, 然而有两点事实将有助 于去理解两者相似性的必然性。第一, 碳磷硫金四 种元素在自然中普遍存在各自的以多原子团聚为特 征的单质态, 如石墨、金刚石, 自然中同样可以找到 的富勒烯、白磷、红磷、多种组成和分子结构的单质 硫、自宏观尺度到微观尺度的自然金. 特别对于金, 迄今还没有从自然中观察到任何金的化合态存在形 式(尽管化学实验室的化合或络合态的金是丰富多 彩的). 第二, 碳磷硫金四种元素在化学上均有较强 的电负性, 尤其金的高电负性数值 ${ }^{[24]}(5.321 \mathrm{eV})$ 是 许多其它常见金属元素所不具有的.

\section{References}

1 Moelvany S W, Cassady C J. J. Phys. Chem., 1990, 94: 2057

2 Liu Jianbo, Xing Xiaopeng, Liu Peng, et al. Int. J. Mass Spectrom., 2000, 202: 261

3 Wang Sufan, Feng Jikang, Cui Meng. Chem. J. Chinese Universities, 1999, 20: 1447 [王素凡, 封继康, 崔 预. 高等 学校化学学报 (Gaodeng Xuexiao Huaxue Xuebao), 1999, 20: 1447 ]

4 Ziemann P J, Castleman J A W. Phys. Rew. B, 1992, 46: 13480

5 Li HY, Ma CS, Bai JL. et al. Acta Phys. -Chim. Sin., 1997, 13: 946 [李海洋, 马晨生, 白吉玲等. 物理化学学报 (Wuli Huaxue Xuebao ), 1997, 13: 946]

6 Shi Y, Zhang N, Gao Z, et al. Chemistry, 1993, 8: 27 [史 扬, 张 南, 高 振等。化学通报 (Huaxue Tongbao), 1993, 8: 27]

7 Chen YQ, Xing XP, Liu P, et al. Chem. J. Chinese Universities, 2000, 20: 743 [陈云清, 邢小鹏, 刘 鹏等. 高等学校化学 学报 (Gaodeng Xuexiao Huaxue Xuebao ), 2000, 20: 743]

8 Zhang N, Yu Z D, Wu X J, et al. J Chem. Soc. Faraday Trans., 1993, 89: 1779

9 Shi Yan, Yu Zhongde, Zhang Nan. J. Chinese Chem. Society, 1995, 42: 455

10 Liu Jianbo, Han Chunying, Zheng Weijun, et al, Int. J. Mass Spectrom., 1999, 189: 147

11 Saunders W A. Phys. Rev. Lett., 1990, 64: 3046

12 Saunders W A. Phys. Rev. A, 1992, 46: 7028

13 Becker S, Dietrich G, Hasse H U, et al. Z. Phys. D, 1994, 30: 341

14 Ziegler J, Dietrich G, Krückeberg S, et al. Int. J. Mass Spectrom., 
2000, 202: 47

15

列

Mass Spectrometry. New York: John Wiley \& Sons., 1987, 241p

16 Liang Handong. Proc. Int. 8th BCEIA, 1999, B: 93

17 Liang Handong. Chinese Science Bulletin, 1999, 44: 1242

18 Bruno W S. Microscopy, Microanalysis, Microstructure, 1992, 3: 119

19 Liang HD. J. Chinese Mass Spectrom. Soc., 1998, 19(1): 84[ [梁 汉东．质谱学报 (Zhipu Xuebao), 1998, 19(1): 84]

20 Chen YQ, Xing XP, Liu P, et al. Chinese J. Chem. Phys., 2000, 13: 257 [陈云清, 邢小鹏, 刘 鹏等。化学物理学报
( Huaxue Wuli Xuebao), 2000, 13: 257]

21 Kroto H W, Heath J R, O'Brien S C, et al., Nature, 1985, 318 162

22 Creasy W R. J. Chem. Phys., 1990, 92: 7223

23 Zeng R, Liu JB, Han CY, Gao Z. Chem. J. Chinese Univ., 2000, 21: 581 [曾 嵘, 刘剑波, 韩春英, 高 振. 高等学校化学学 报 (Gaodeng Xuexiao Huaxue Xиebao ), 2000, 21: 581]

24 Yu D, Chen ZD, Wang F, Li SZ., Acta Phys. -Chim. Sin., 2001, 17 (1): 15 [喻 典, 陈志达, 王 繁, 李述周. 物理化 学学报 (Wuli Huaxue Xuebao), 2001, 17 (1): 15]

\title{
Compositional and Constitutional Characterization of Au-S Cluster lons*
}

Liang Han-Dong

( The Key Laboratory of Coal Resources, China University of Mining and Technology-Beijing, Beijing

$100083)$

Liu Dun-Yi

( Isotope Geology Laboratory, Chinese Academy of Geoscience, Beijing 100037)

\begin{abstract}
An observation is demonstrated that $\mathrm{Au} / \mathrm{S}$ target, triggered by gallium ion beam $\left({ }^{69} \mathrm{Ga}^{+}\right)$under experiment of high performance secondary ion mass spectrometry (TOF SIMS), does emit a considerable species of cluster negative ions with general formula of $\mathrm{Au}_{1-15} \mathrm{~S}_{0-5}$ (see Fig. 1 and Table 1). Among them, a series of cluster ions with compositions of $\mathrm{Au}_{1-15} \mathrm{~S}_{1-5}$ is recognized as $\mathrm{Au}-\mathrm{S}$ binary cluster ions, while other series with $\mathrm{Au}_{1-15}$ belongs to mono-cluster ions of pure gold, appearing in strong odd-predominance of their intensities with number of gold atom (see Fig. 2). The Au-S cluster ions can be characterized, in some aspects, by existence of the odd-predominance with magnitudes of gold atom (see Fig. 3), inherited from the Au mono-clusters; by lack of stoichiometric feature, showing that gold atoms range from 1 to 15 but sulfur atoms are always limited to 5 (see Table 1); by void of predominance as compositions as $\mathrm{Me}_{n} \mathrm{~S}_{n}$ (for example, $\mathrm{Cu}_{n} \mathrm{~S}_{n}$, see Table 2) or $\mathrm{Me}_{n} \mathrm{~N}_{n}$ whose priority has been proved as an important feature prevailing in other binary cluster ions of metals (Me) plus sulfur (S) or other non-metals $(\mathrm{N})$; and by presence of special profiles as similar as those of C-P binary cluster ions, a series of typical non-metal-binary ones (see, Ref. 23). These facts suggest that the speciation of Au-S cluster ions probably comes from a constitution of relatively intact Au mono-clusters, and thus imply in gaseous-phase mechanism that a binary aggregation between Au mono-clusters and $\mathrm{S}$ mono-clusters or the confined poly-sulfur units might contribute to the formation of the Au-S binary cluster ions. The aggregation may be based on covalent bindings between the end groups of the two clusters, or on a non-covalent interaction between the clusters, such as electrostatic gravitation between hetero-atoms or hetero-molecules, but its true mechanism remains to be resolved in future work.
\end{abstract}

Keywords: Au-S binary clusteri ons, Au mono-cluster, Five-sulfur units, Aggregation, Cluster negative ions, Compositional characterization, TOF SIMS 